\title{
Propriedades físicas e tecnológicas de farinha de trigo tratada com terra diatomácea
}

\author{
Technological and physical properties of wheat flour treated with diatomaceous earth
}

\author{
Janete Deliberali Freo ${ }^{\mathrm{I}}$ Lidiane Borges Dias de Moraes ${ }^{\mathrm{I}}$ Rosana Colussi $^{\mathrm{II}}$ Juliane Mossmann ${ }^{\mathrm{II}}$ \\ Moacir Cardoso Elias ${ }^{\text {III }}$ Luiz Carlos Gutkoski ${ }^{\text {IV }}$
}

\section{RESUMO}

Embora exista grande número de trabalhos sobre a aplicação de terra diatomácea no controle de insetos em grãos de trigo, as informações sobre o resíduo que permanece na farinha após a moagem, mesmo com retirada parcial na etapa de limpeza, e se este altera a qualidade tecnológica, são restritas. $O$ objetivo deste estudo foi avaliar as propriedades físicas e tecnológicas de farinha de trigo tratada com diferentes terras diatomáceas. As terras diatomáceas de marcas comerciais, codificadas como TD1 e TD2 e a farinha de trigo tipo I foram adquiridas no mercado local. A TD1 e TD2 foram adicionadas nas doses zero, 0,5, 1,0 e 2,0 $\mathrm{g} \mathrm{kg}^{-1}$ de farinha de trigo, homogeneizadas em misturador e realizadas as análises número de queda, alveografia, farinografia, cor e teste de panificação experimental. O experimento foi conduzido em delineamento inteiramente casualizado em arranjo fatorial $2 \times 4$, os resultados submetidos à análise de variância (ANOVA) e nos modelos significativos, as médias comparadas entre si pelo teste de Tukey a 5\% de significância. A terra diatomácea adicionada na quantidade de até $2 \mathrm{~g} \mathrm{kg-1}$ afeta as propriedades físicas e funcionais da farinha de trigo. O alveógrafo é efetivo para predizer alterações nas propriedades físicas de farinha de trigo tratada com terra diatomácea. As alterações mais acentuadas nas propriedades físicas da farinha de trigo são do componente $L^{*}$ (luminosidade) de cor e força geral de glúten $(W)$, enquanto que nas tecnológicas o escore de pontos e componente $L^{*}$ de cor do miolo.

Palavras-chave: Triticum aestivum, alveografia, panificação, escore de pontos.

\section{ABSTRACT}

Although there are many studies on the application of diatomaceous earth for insect control in wheat grains, the information about the residue that remains in the flour after grinding, even with partial removal in the cleaning step, and if this changes the technological quality are restricted. The objective of this study was to evaluate the physical and functional properties of wheat flour treated with diatomaceous earth. Diatomaceous earth of two commercial brands codified as DE1 and DE2, were used. Wheat flour type I was purchased at the local market. DE1 and DE2 were added in doses zero, $0.5,1.0$ and $2.0 \mathrm{~g} \mathrm{~kg}^{-1}$ of wheat flour, homogenized in a blender and the analysis of falling number, alveography, farinograph, color analysis and experimental baking test were carried out. The results were analyzed by Analysis of variance and comparisons of means by Tukey's test at 5\% significance level. The diatomaceous earth added in doses up to $2 \mathrm{~g} \mathrm{~kg}^{-1}$ directly into the flour affects the physical and functional properties. The alveograph is effective to predict changes in physical properties of wheat flour. The changes more pronounced in wheat flour physical properties are color component lightness and gluten strength $(W)$, while in the baking test were points score and crumb color component lightness.

Key words: Triticum aestivum, alveography, baking, points score.

IPrograma de Pós-graduação em Ciência e Tecnologia Agroindustrial, Faculdade de Agronomia Eliseu Maciel, Universidade Federal de Pelotas (UFPel), Pelotas, RS, Brasil.

${ }^{\text {IIC }}$ urso de Engenharia de Alimentos, Universidade de Passo Fundo (UPF), Passo Fundo, RS, Brasil.

IIIDepartamento de Ciência e Tecnologia Agroindustrial, UFPel Pelotas, RS, Brasil.

${ }^{\text {IV }}$ Faculdade de Agronomia e Medicina Veterinária, UPF, Campus universitário, CP 611, 99001-970, Passo Fundo, RS, Brasil. Email: gutkoski@upf.br. Autor para correspondência. 


\section{INTRODUÇÃO}

A qualidade de grãos e farinhas de cereais é determinada por uma variedade de características que assumem diferentes significados dependendo da designação de uso ou tipo de produto. Estas características podem ser divididas em físicas, químicas, enzimáticas e reológicas. A avaliação reológica da farinha é de fundamental importância para a indústria de panificação, ajudando a predizer as características de processamento da massa e a qualidade dos produtos finais, sendo parte de um conjunto de análises, no qual necessariamente deve estar incluído o teste de panificação (GUTKOSKI \& JACOBSEN NETO, 2002; ABID et al., 2009), quando a farinha de trigo destina-se a esta forma de produto final.

A terra diatomácea é um sedimento amorfo, originado a partir de carapaças de organismos unicelulares vegetais tais como algas microscópicas aquáticas, marinhas e lacustres, normalmente denominados pós inertes. Por apresentarem natureza silicosa, as frústulas desenvolvem-se nas camadas geológicas da crosta terrestre. A terra diatomácea é um material leve e de baixa massa específica aparente, coloração variável desde o branco ao cinza escuro. Este material é constituído principalmente por óxido de silício (80\% a 93\%), argilominerais, material orgânico, hidróxidos, areia quartzosa, carbonatos de cálcio e de magnésio (KORUNIC et al., 1998; VARDEMAN et al., 2007).

Os depósitos fossilizados de terra diatomácea são recolhidos e processados para uso comercial por secagem, trituração e moagem em pó fino. A terra diatomácea é um produto atóxico, utilizado no controle de pragas de grãos armazenados e não promove a resistência em insetos (LORINI et al., 2003). O modo de ação ocorre pela desidratação ou dessecação, uma vez que partículas do pó aderem ao corpo dos insetos removendo a cera epicuticular, causando abrasão no tegumento e perda excessiva de água (VARDEMAN et al., 2007).

A aplicação de terra diatomácea em grãos tem apresentado problemas como alterações em propriedades físicas e mecânicas, reduzindo escoabilidade e densidade, com presença visível de resíduos. Outro problema é o efeito da abrasividade em equipamentos e o aumento do tempo de moagem dos grãos de trigo tratados (MIRANDA et al., 1999).

Embora exista grande número de trabalhos sobre a aplicação de terra diatomácea no controle de insetos em grãos de trigo, as informações sobre o resíduo que permanece na farinha após a moagem, mesmo com retirada parcial na etapa de limpeza, e se este altera a qualidade tecnológica, são restritas. Objetivou-se, com o trabalho, avaliar propriedades físicas e tecnológicas de farinha de trigo tratada com terra diatomácea de duas marcas comerciais, realizada por meio das determinações número de queda, alveografia, farinografia, cor e teste de panificação.

\section{MATERIAL E MÉTODOS}

Para a realização do trabalho foram utilizadas terras diatomáceas de duas marcas comerciais TD1 e TD2. A TD1 foi adquirida em saco de 5kg, lote no $274-$ 09-520, fabricada em agosto de 2008 com validade até agosto de 2013. A TD2 foi adquirida em saco de $20 \mathrm{~kg}$, lote no 372-08-700, fabricada em outubro de 2008 com validade até outubro de 2013. A farinha de trigo tipo I foi adquirida no mercado local, em quantidade de $15 \mathrm{~kg}$. As terras diatomáceas TD1 e TD2 foram adicionadas nas doses zero, 0,5, 1,0 e 2,0 $\mathrm{g} \mathrm{kg}^{-1}$ de farinha de trigo, realizada a homogeneização em misturador marca Kitchen Aid $^{\circledR}$, modelo K5SSWH2, na velocidade média pelo tempo de cinco minutos. O experimento foi conduzido em delineamento inteiramente casualizado em arranjo fatorial $2 \times 4$, ou seja, duas marcas de terra diatomácea e quatro doses, sendo as análises realizadas no mínimo em duplicata, no Laboratório de Cereais do Centro de Pesquisa em Alimentação (CEPA) da Universidade de Passo Fundo (UPF).

O número de queda da farinha de trigo foi determinado em aparelho Falling Number, marca Perten Instruments ${ }^{\circledR}$, modelo Fungal 1500, realizado de acordo com o método 56-81B da AACC (2000), utilizando massa de sete gramas de amostra, corrigido para 14\% de umidade, conforme tabela de correção de massa do manual do aparelho, bem como correção de altitude devido o laboratório estar localizado a 710m, através da ativação da função corrigir altura do aparelho.

As características viscoelásticas foram determinadas no aparelho alveógrafo, marca Chopin ${ }^{\circledR}$ (modelo NG, França), realizado de acordo com o método $\mathrm{n}^{\circ}$ 54-30 da AACC (1999), por meio da pesagem de $250 \mathrm{~g}$ de farinha e volume de 129,4mL de água, corrigido na base de $14 \%$ de umidade. Os parâmetros considerados foram tenacidade $(\mathrm{P})$, que mede a sobrepressão máxima exercida na expansão da massa ( $\mathrm{mm}$ ); extensibilidade (L), que mede o comprimento da curva ( $\mathrm{mm}$ ) e força geral do glúten (W), que corresponde ao trabalho mecânico necessário para expandir a bolha até a ruptura, expressa em $10^{-4} \mathrm{~J}$.

A cor das amostras de farinha de trigo e de miolo do pão foram determinadas em aparelho espectrofotômetro marca HunterLab ${ }^{\circledR}$, (modelo 
ColorQuest II Sphere, Inglaterra), com sensor ótico geométrico de esfera. O aparelho foi calibrado com cerâmica e realizada a leitura por reflexão e ângulo de observação de $2^{\circ}$, iluminante principal D75 e iluminante secundário D65. No sistema Hunter de cor, corrigido pela CIELAB (International Comission on Illumination), os valores $L^{*}$ (luminosidade) variam entre zero (preto) e 100 (branco), -a* (verde) até $+a^{*}$ (vermelho), e -b* (azul) até $+\mathrm{b}^{*}$ (amarelo).

A capacidade de absorção de água e as propriedades de mistura foram determinadas no aparelho Promilógrafo, marca Max Egger ${ }^{\circledR}$, (modelo T6, Áustria), realizado de acordo com o método no 54-21 da AACC (1995). Os parâmetros avaliados foram absorção de água, que indica a capacidade de intumescimento do glúten e o teor de amido danificado; tempo de desenvolvimento, que corresponde ao intervalo decorrente desde a primeira adição de água até o ponto de máxima consistência (pico); estabilidade, definida como a diferença de tempo entre o ponto em que o topo da curva intercepta a linha média de 500UP e o ponto da curva que deixa a linha.

O teste de panificação experimental foi realizado de acordo com o método nํㅜㅅ-80B da AACC (1999), com adaptações propostas por GUTKOSKI \& JACOBSEN NETO (2002), sendo utilizada farinha (100\%), gordura vegetal hidrogenada (3\%), sal refinado (1,75\%), ácido ascórbico (0,01\%), açúcar (5\%), fermento biológico (3\%) e água a $4^{\circ} \mathrm{C}$, adicionada de acordo com a absorção no promilógrafo. Os ingredientes foram misturados na misturadora marca Kitchen Aid ${ }^{\circledR}$ (modelo K5SSWH2, EUA), na velocidade média por sete minutos. O fermento biológico foi adicionado e realizada a mistura por mais seis minutos. A massa foi retirada da misturadora, dividida em porções de $150 \mathrm{~g}$, colocada em formas de tamanho padrão e deixada em descanso por dez minutos. A fermentação foi realizada na câmara (marca Multipão ${ }^{\circledR}$, Brasil), regulada na temperatura de $30 \pm 1^{\circ} \mathrm{C}$ e umidade relativa de $80 \%$. O cozimento foi realizado no forno marca Labor Instruments ${ }^{\circledR}$ (modelo QA 226, Hungria), regulado na temperatura de $220^{\circ} \mathrm{C}$ por 18 minutos e após uma hora realizadas as análises nos pães tipo forma.

A avaliação do volume dos pães foi determinada em aparelho (marca Vondel ${ }^{\circledR}$, Brasil), pelo deslocamento de sementes de canola e o volume específico calculado pela relação entre o volume do pão assado e a sua massa, obtida por pesagem em balança eletrônica Mark ${ }^{\circledR}$ (modelo 3100, EUA), com precisão de $0,01 \mathrm{~g}$. A determinação do volume específico foi realizada uma hora após o cozimento dos pães, com três repetições e os resultados expressos em $\mathrm{cm}^{3} \mathrm{~g}^{-1}$. A avaliação do escore de pontos dos pães foi realizada utilizando a escala de pontos para as características cor da crosta, forma e simetria, características da crosta, aspectos de quebra da crosta, textura do miolo, cor do miolo, aroma e sabor, conferindo-lhes, a partir desses resultados, um valor (escore de pontos) com pontuação máxima de 100, realizado de acordo com a metodologia proposta por FERREIRA (2002).

Os resultados foram submetidos à análise de variância (ANOVA) e, nos modelos significativos, as médias comparadas entre si pelo teste de Tukey a $5 \%$ de significância. O processamento de dados e a análise estatística foram realizados com o programa estatístico SAS ${ }^{\circledR}$ (SAS INSTITUTE, 1985).

\section{RESULTADOS E DISCUSSÃO}

A umidade das amostras de farinha de trigo não variou significativamente $(\mathrm{P} \geq 0,05)$ entre as doses empregadas, sendo significativamente superior em relação à marca TD2 empregada, apresentado valores de 13,20\%, em média (Tabela 1). Nas doses 0,5 e 1,0g $\mathrm{kg}^{-1}$, os valores de umidade foram significativos $(\mathrm{P}<0,05)$ para TD1. O teor de umidade máximo da farinha permitido pela legislação é de 15\%, estando os valores, portanto, abaixo deste limite (BRASIL, 2005). A umidade deve ser monitorada por ser um dos principais fatores de aceleração de reações químicas e enzimáticas da farinha de trigo (GUTKOSKI et al., 2002).

A cor da farinha é avaliada pelas medidas de luminosidade $\left(\mathrm{L}^{*}\right)$ e coordenada de cromaticidade de amarelo $\left(+b^{*}\right)$. A luminosidade é afetada pelo conteúdo de farelo ou material estranho, enquanto a intensidade de amarelo está relacionada com a quantidade de pigmentos presentes na farinha de trigo (COULTATE, 2004). O componente $L^{*}$ de cor diminuiu significativamente ( $\mathrm{P} \leq 0,05)$ com o aumento de dose e também entre as marcas de terra diatomácea. Entre as marcas estudadas, a TD2 apresentou intensidade do componente $\mathrm{L}^{*}$ significativamente inferior a TD1. Quanto às coordenadas de cromaticidade $a^{*}$ e b*, foi verificada variação significativa entre doses de TD2 e entre TD1 e TD2. A TD2 mostrou redução da coordenada de cromaticidade do componente verde ($\mathrm{a}^{*}$ ) e aumento a coordenada de cromaticidade componente $+b^{*}$ com o aumento de dose, o que resultou em farinha de trigo mais escura (Tabela 1 ).

A determinação do número de queda em farinha de trigo tem por finalidade verificar a atividade das enzimas amilolíticas da farinha. O teste avalia de forma indireta o grau de germinação de trigo na espiga e a capacidade de fermentação da massa, pois, quanto menor o valor encontrado, maior a atividade amilolítica da farinha (CARNEIRO et al., 2005). O número de queda 
Tabela 1 - Valores médios ${ }^{1}$ umidade, componentes $\mathrm{L}^{*}, \mathrm{a}^{*}$ e b*, número de queda (NQ), força de glúten (W), elasticidade (P), extensibilidade (L), absorção de água (AA) e estabilidade (E) de amostras de farinha de trigo tratadas com terras diatomáceas de duas marcas comerciais (TD1 e TD2).

\begin{tabular}{|c|c|c|c|c|c|c|c|c|}
\hline \multirow[b]{3}{*}{ Determinação } & \multicolumn{8}{|c|}{ Tratamento } \\
\hline & \multicolumn{4}{|c|}{ 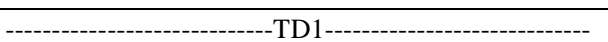 } & \multicolumn{4}{|c|}{-------------------------------TD2------------------------------ } \\
\hline & Zero & 0,5 & 1,0 & 2,0 & Zero & 0,5 & 1,0 & 2,0 \\
\hline Umidade (\%) & $13,04 \mathrm{aA}$ & $13,58 \mathrm{aA}$ & $13,30 \mathrm{aA}$ & $13,25 \mathrm{aA}$ & $13,04 \mathrm{aA}$ & $12,83 a \mathrm{aB}$ & $13,19 \mathrm{aB}$ & $13,18 \mathrm{aA}$ \\
\hline Componente L* & $93,64 \mathrm{aA}$ & 93,37bA & $93,27 \mathrm{bA}$ & 93,13bA & $93,64 \mathrm{aA}$ & $92,88 \mathrm{bB}$ & $92,78 \mathrm{cB}$ & $92,67 \mathrm{~dB}$ \\
\hline Componente $\mathrm{a}^{*}$ & $-0,97 \mathrm{aA}$ & $-0,96 a B$ & $-0,94 \mathrm{aA}$ & $-0,93 \mathrm{aB}$ & $-0,97 \mathrm{cA}$ & $-0,92 b A$ & $-0,92 b A$ & $-0,87 \mathrm{aA}$ \\
\hline Componente b* & $7,82 \mathrm{aA}$ & 7,94aA & $7,90 \mathrm{aA}$ & $7,87 \mathrm{aA}$ & 7,82aA & $7,72 \mathrm{bA}$ & 7,69bB & 7,67bB \\
\hline NQ (s) & $311 \mathrm{aA}$ & 331aA & 301aA & $314 \mathrm{aA}$ & $311 \mathrm{aA}$ & $306 a A$ & 313aA & $312 \mathrm{aA}$ \\
\hline $\mathrm{W}\left(10^{-4} \mathrm{~J}\right)$ & $234 \mathrm{aA}$ & $225 \mathrm{bA}$ & 221bA & 198cA & $234 \mathrm{aA}$ & $200 \mathrm{bB}$ & $166 \mathrm{cB}$ & $164 \mathrm{cB}$ \\
\hline $\mathrm{P}$ & $92,5 \mathrm{aA}$ & $85,5 \mathrm{bA}$ & $88,0 \mathrm{abB}$ & $78,0 \mathrm{cB}$ & $92,5 \mathrm{bA}$ & $79 \mathrm{cB}$ & 98abA & $101,5 \mathrm{aA}$ \\
\hline $\mathrm{L}$ & $61,5 \mathrm{aA}$ & $64,5 \mathrm{aA}$ & $60,5 \mathrm{aA}$ & $62,5 \mathrm{aA}$ & $61,5 \mathrm{aA}$ & $58,5 \mathrm{aB}$ & $36 \mathrm{bB}$ & $34,5 b B$ \\
\hline $\mathrm{AA}(\mathrm{mL})$ & $55,00 \mathrm{aA}$ & $55,00 \mathrm{aA}$ & $55,15 \mathrm{aA}$ & $55,55 \mathrm{aA}$ & $55 a A$ & $55,05 \mathrm{Aa}$ & $54,4 \mathrm{abB}$ & $53,75 b B$ \\
\hline $\mathrm{E}(\mathrm{min})$ & $19,60 \mathrm{aA}$ & $17,00 \mathrm{bB}$ & $17,00 \mathrm{bB}$ & 17,50bB & 19,6bA & 20,4abA & $22 \mathrm{aA}$ & $22,25 \mathrm{aA}$ \\
\hline
\end{tabular}

${ }^{1}$ Médias seguidas das mesmas letras minúsculas e maiúsculas na linha não diferem estatisticamente pelo teste de Tukey a 5\% de probabilidade quanto a doses (de zero a 2,0) e marca de terra diatomácea (TD1 e TD2), respectivamente.

Em que: L* (luminosidade); TD1 e TD2 são duas marcas comerciais de terra diatomácea; W, P e L são parâmetros da alveografia e AA e E, parâmetros da farinografia.

da farinha apresentou valores médios de 310 segundos (Tabela 1), não variando significativamente entre si $(\mathrm{P} \geq 0,05)$. Os resultados deste trabalho estão de acordo com o obtido por KORUNIC et al. (1996), que não verificou diferenças significativas entre farinhas de amostras de trigo tratado com $300 \mathrm{mg} \mathrm{kg}^{-1}$ de terra diatomácea para número de queda.

A alveografia é um teste reológico que determina a força de glúten (W), sendo utilizada para avaliar a maior ou menor capacidade da farinha sofrer um tratamento mecânico ao ser misturada com água. É associada também à capacidade de absorção de água pelas proteínas, que contribuem na retenção do gás carbônico dos produtos de panificação (DOBRASZCZYK \& MORGENSTERN, 2003). A força de glúten (W) diminuiu com o aumento das doses de terra diatomácea, sendo significativamente superior nas amostras tratadas com TD2. Os valores de tenacidade (P) apresentaram variações significativas $(p \leq 0,05)$ com o aumento das doses de terra diatomácea, sendo superior em TD2. Para os valores de extensibilidade (L), os resultados não variaram significativamente entre si $(P \geq 0,05)$ em TD1, ocorrendo redução para TD2 (Tabela 1). Segundo MIRANDA et al. (1999) os pós inertes utilizados em grãos de trigo em doses de $2 \mathrm{~kg}$ por tonelada não provocaram alterações na qualidade da farinha, porém no trigo foi verificada redução do peso de hectolitro e aumentou do tempo de moagem dos grãos tratados. Os autores indicam que o emprego desse produto em trigo armazenado deve ser informado no momento da comercialização, pois afeta a classificação comercial do grão. No presente trabalho, foi verificado efeito significativo de dose nas propriedades viscoelásticas da massa, indicando a necessidade de quantificar o teor residual de terra diatomácea presente na farinha após a moagem de trigo tratado.

A farinografia é um teste completo e sensível para avaliação da qualidade da absorção de água e mistura da massa de farinha de trigo (GUARIENTI, 1996). Na farinha tratada com TD1, não foi verificada variação na absorção de água (AA), enquanto para TD2 ocorreu diminuição de AA com a elevação da dose de terra diatomácea. Para amostras tratadas com TD1, foi verificada redução na estabilidade (E) enquanto que, em TD2, esta aumentou com a elevação da quantidade de terra diatomácea (Tabela 1). Os maiores valores de E ocorreram nas amostras de farinha de trigo tratado com TD2, não sendo verificada relação com os resultados de alveografia, em especial à força de glúten. Os resultados deste trabalho mostraram que o alveógrafo foi mais efetivo para avaliar as propriedades físicas e predizer o uso final da farinha de trigo tratada com terra diatomácea em comparação com o aparelho farinógrafo.

Conforme KORUNIC et al. (1996), farinha de amostras de trigo tratado com 300 $\mathrm{mg} \mathrm{kg}^{-1}$ de terra diatomácea apresentou maior estabilidade na mistura e tempo de desenvolvimento da massa, quando comparado com farinha tratada com $50 \mathrm{mg} \mathrm{kg}^{-1}$. No entanto, o aumento da estabilidade da massa não se refletiu no aumento de volume específico de pão no teste de panificação. 
A obtenção de pão de qualidade requer desempenho adequado da farinha em relação ao desenvolvimento da massa. Isso envolve produção adequada de gás, propriedades viscoelásticas da massa e capacidade de retenção de gás. O desenvolvimento da massa e o aspecto final do pão dependem da qualidade e da quantidade de ingredientes e do emprego de tecnologia adequada de fabricação (MONDAL \& DATTA, 2008).

No teste de panificação experimental, foram determinados volume específico, características externas (cor da crosta, forma e características da crosta), características internas (porosidade, cor do miolo e textura do miolo), aroma e sabor dos pães, obtendo-se o escore de pontos (Tabela 2). O volume específico (VE) não variou significativamente nas amostras tratadas com TD1 e foi verificada uma redução no VE com o aumento de dose em TD2. Este comportamento está em acordo com o verificado nas propriedades físicas da farinha de trigo, avaliada pelo aparelho alveógrafo, que indicou redução de propriedades viscoelásticas da massa com o aumento de dose em TD2. Nas avaliações de características externas (CE) dos pães, não foram verificadas diferenças significativas. Porém, para características internas (CI) dos pães, foi atribuída menor pontuação com o aumento de dose de terra diatomácea. Este trabalho está em acordo com o realizado por DESMARCHELIER \& DINES (1987), pois verificaram que bolos elaborados com farinha de trigo tratada com $100 \mathrm{mg} \mathrm{kg}^{-1}$ de terra diatomácea apresentaram textura mais grossa e volume de $7 \%$ a $10 \%$ menor que os elaborados com farinha de trigo não tratada.

Para aroma e sabor, foi verificada redução significativa $(\mathrm{P} \leq 0,05)$ com o aumento da dose de terra diatomácea, porém sem diferir significativamente entre as marcas de terra diatomácea estudadas. Os avaliadores descreveram, na avaliação dos pães, a presença de sabor adstringente crescente com o aumento da dose de terra diatomácea na farinha de trigo.

O escore de pontos dos pães variou significativamente com o aumento de dose de TD1 e TD2. Os pães elaborados com farinha sem adição de terra diatomácea foram classificados como de boa qualidade, com valor de 88 no escore de pontos. A adição de terra diatomácea na farinha de trigo reduziu o escore de pontos dos pães, com classificação regular quando empregado $2 \mathrm{~g} \mathrm{~kg}^{-1} \mathrm{em}$ TD1 e em todas as doses em TD2, sendo verificados valores inferiores a 81 (FERREIRA, 2002). Os pães elaborados com TD2 apresentaram escore de pontos inferior aos pães elaborados com farinha de trigo tratada com TD1. Esse comportamento está em acordo com os resultados de alveografia e da intensidade de cor do componente $\mathrm{L}^{*}$ das amostras de farinha de trigo tratadas com terra diatomácea. A cor do miolo dos pães avaliado pela intensidade de cor do componente $\mathrm{L}^{*}$ diminuiu com o aumento da dose de terra diatomácea, variando significativamente também entre as marcas TD1 e TD2 estudadas. Os resultados de cor do componente $L^{*}$ do miolo dos pães apresentaram relação com os obtidos na farinha de trigo, afetando negativamente a qualidade final do produto.

\section{CONCLUSÃO}

A terra diatomácea adicionada na quantidade de até $2 \mathrm{~g} \mathrm{~kg}^{-1}$ afeta as propriedades físicas e funcionais da farinha de trigo. Na farinha de trigo tratada com TD2, as alterações são mais acentuadas. $\mathrm{O}$ alveógrafo é mais efetivo que o farinógrafo para predizer

Tabela 2 - Valores médios ${ }^{1}$ volume específico (Vol. esp.), características externas (C E), características internas (C I), aroma e sabor, escore de pontos e cor do miolo $\left(\mathrm{L}^{*}\right)$ de amostras de farinha de trigo tratadas com duas marcas comerciais de terra diatomácea (TD1 e TD2).

\begin{tabular}{|c|c|c|c|c|c|c|c|c|}
\hline \multirow[b]{3}{*}{ Determinação } & \multicolumn{8}{|c|}{ Tratamento } \\
\hline & \multicolumn{4}{|c|}{ 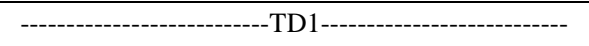 } & \multicolumn{4}{|c|}{----------------------------TD2--------------------------- } \\
\hline & Zero & 0,5 & 1,0 & 2,0 & Zero & 0,5 & 1,0 & 2,0 \\
\hline Vol. esp. $\left(\mathrm{cm}^{3} \mathrm{~g}^{-1}\right)$ & $14 \mathrm{aA}$ & $14 \mathrm{aA}$ & $14 \mathrm{aA}$ & $14 \mathrm{aA}$ & $14 \mathrm{aA}$ & $10 \mathrm{bB}$ & $10 \mathrm{bB}$ & 10bB \\
\hline C E & $26,10 \mathrm{aA}$ & $26,06 \mathrm{aA}$ & $25,80 \mathrm{aA}$ & $25,56 \mathrm{aA}$ & $26,10 \mathrm{aA}$ & 25,83aA & $25,83 a A$ & $25,83 \mathrm{aA}$ \\
\hline C I & 28,03aA & 26,56bA & $25,53 \mathrm{bA}$ & $23,76 \mathrm{cB}$ & 28,03aA & 26,26bA & 25,86bA & $25,80 \mathrm{bA}$ \\
\hline Aroma e sabor & 19,83aA & $18,06 \mathrm{bA}$ & 16,70cA & $14,00 \mathrm{~dB}$ & $19,83 a A$ & $18,00 \mathrm{bA}$ & $16,50 \mathrm{cA}$ & $15,00 \mathrm{dA}$ \\
\hline Escore (0 a 100) & $87,93 a A$ & $85,10 \mathrm{bA}$ & $82,03 \mathrm{cA}$ & $77,33 \mathrm{dA}$ & $87,93 a A$ & $80,10 \mathrm{bB}$ & $78,20 \mathrm{cB}$ & 76,63dA \\
\hline Cor miolo ( $\left.\mathrm{L}^{*}\right)$ & 77,10aA & $76,54 \mathrm{bA}$ & $74,36 \mathrm{cB}$ & $73,30 \mathrm{~dB}$ & 77,10aA & 76,45bA & 75,19cA & $74,56 \mathrm{dA}$ \\
\hline
\end{tabular}

${ }^{1}$ Médias seguidas das mesmas letras minúsculas e maiúsculas na linha não diferem estatisticamente pelo teste de Tukey a 5\% de probabilidade quanto a doses (de zero a 2,0) e marca de terra diatomácea (TD1 e TD2), respectivamente. 
alterações nas propriedades físicas de farinha de trigo tratada com terra diatomácea.

As alterações mais acentuadas nas propriedades físicas da farinha de trigo são do componente $\mathrm{L}^{*}$ (luminosidade) de cor e força geral de glúten (W), enquanto que nas propriedades tecnológicas o escore de pontos e componente $\mathrm{L}^{*}$ do miolo.

\section{AGRADECIMENTOS}

Ao Conselho Nacional de Desenvolvimento Científico e Tecnológico (CNPq), pelo apoio financeiro. Às empresas GP Representações e Protection Comércio e Representações de Insumos Ltda., pelo fornecimento de amostras de terra diatomácea.

\section{REFERÊNCIAS}

AACC- American Association of Cereal Chemists. Approved methods. 10.ed. Saint Paul: AACC, 2000. Paginação irregular.

BID, H. et al. Suitability of different wheat varieties grown in NWFP for bread making and effects of falling number on storage. Journal of Nutrition, v.8, n.5, p.616-619, 2009. Disponível em: <http://docsdrive.com/pdfs/ansinet/pjn/2009/616-619.pdf> . Acesso em: 15 abr. 2009.

BRASIL. Ministério da Agricultura, Pecuária e Abastecimento. Instrução normativa n.8, 03 jun. 2005. Regulamento Técnico de Identidade e Qualidade da Farinha de Trigo. Diário Oficial da República Federativa do Brasil, Brasília, DF, 03 jun. 2005, Seção 1, n.105, 9.91.

CARNEIRO, L.M.T.A. et al. Diferentes épocas de colheita, secagem e armazenamento na qualidade de grãos de trigo comum e duro. Bragantia, v.64, n.1, p.127-137, 2005.

COULTATE, T.P. Alimentos: a química de seus componentes. 3.ed. Porto Alegre: Artmed, 2004. 368p.

DESMARCHELIER, J.M.; DINES, J.C. Dryacide treatment of stored wheat: its efficacy against insects, and after processing. Australian Journal of Experimental Agriculture, v.27, n.2, p.309-312, 1987.

DOBRASZCZYK, B.J.; MORGENSTERN, M.P. Rheology and the breadmaking process. Journal of Cereal Science, v.38, n.2, p.229-245, 2003. Disponível em: <http://www.sciencedirect.com/ science>. Acesso em: 20 nov. 2009. doi:10.1016/S07335210(03)00059-6.

FERREIRA, S.M.R. Controle da qualidade em sistemas de alimentação coletiva I. São Paulo: Varela, 2002. 173p.

GUARIENTI, E.M. Qualidade industrial de trigo. 2.ed. Passo Fundo: Embrapa, 1996. 33p.

GUTKOSKI, L.C. et al. Correlação entre o teor de proteínas em grãos de trigo e a qualidade industrial das farinhas. Boletim Ceppa, v.20, n. 1, p.29-40, 2002.

GUTKOSKI, L.C.; JACOBSEN NETO, R. Procedimento para teste laboratorial de panificação - pão de forma. Ciência Rural, v.32, n.5, p.873-879, 2002.

KORUNIC, Z. et al. Grain bulk density as affected by diatomaceous earth and application method. Postharvest Biology and Technology, v.13, n.1, p.81-89, 1998. Disponível em: <http://www.sciencedirect.com/science>. Acesso em: 20 nov. 2009. doi:10.1016/S0925-5214(97)00076-8.

KORUNIC, Z. et al. The effect of diatomaceous earth on grain quality. Postharvest Biology and Technology, v.9, n.3, p.373387, 1996. Disponível em: <http://www.sciencedirect.com/ science>. Acesso em: 20 nov. 2009. doi:10.1016/S09255214(96)00038-5.

LORINI, I. et al. Tratamento de sementes armazenadas com pós inertes a base de terra diatomácea. Revista Grãos Brasil, v.2, n.12, p.6-7, 2003.

MIRANDA, M.Z. et al. Efeito de pós inertes usados no controle de insetos de grãos armazenados sobre características de qualidade de trigo. In REUNIÃO NACIONAL DE PESQUISA DE TRIGO, 18., 1999, Passo Fundo, RS. Anais... Passo Fundo: Embrapa Trigo, 1999. V.1, p.406-411.

MONDAL, A.; DATTA, A.K. Bread baking - a review. Journal of Food Engineering, v.86, n.4, p.465-474, 2008. Disponível em: <http://top25.sciencedirect.com/subject/chemical-engineering/ 5/journal/journal-of-food-engineering/02608774/archive/15>. Acesso em: 19 mar. 2009. doi:10.1016/j.jfoodeng.2007.11.014.

SAS INSTITUTE. User's guide: statistics. 5.ed. Cary, 1985. 965p.

VARDEMAN, E.A. et al. Efficacy of surface applications with diatomaceous earth to control Rhyzopertha dominica (F.) (Coleoptera: Bostrichidae) in stored wheat. Journal of Stored Products Research, v.43, n.4, p.335-341, 2007. Disponível em: <http://linkinghub.elsevier.com/retrieve/pii/S0022474X06000701>. Acesso em: 19 mar. 2009. doi:10.1016/j.jspr.2006.08.003. 